\title{
Salivary Interleukin 1-beta levels and clinical periodontal parameters in habitual naswar users and non-users
}

\author{
Zaeem Arif Abbasi ${ }^{1}$, Naila Irum Hadi², \\ Adnan Mustafa Zubairi ${ }^{3}$, Mervyn Hosein ${ }^{4}$
}

\begin{abstract}
Objectives: Aim of our study was to assess the expression of salivary Interleukin 1-beta (IL-1B) and clinical periodontal parameters in naswar users and non-users (controls).

Methods: Eighty four individuals (forty-two naswar users and forty-two controls) were included in the study which was conducted between August 2017 and May 2018. Salivary IL-1B levels, plaque index (PI), bleeding on probing (BOP), probing depth (PD) and clinical attachment loss (CAL) was assessed in all the participants.

Results: PD of $4 \mathrm{~mm}(\mathrm{p}<0.05)$, PD of $5-6 \mathrm{~mm}(\mathrm{p}<0.05)$, CAL $(p<0.001)$ and levels of salivary IL1B $(\mathrm{p}<0.05)$ were significantly higher among naswar users as compared to controls while $\mathrm{PI}, \mathrm{BOP}$ and number of missing teeth showed no significant difference among the two groups ( $p>0.05)$.

Conclusion: Periodontal inflammatory conditions were worse and salivary IL-1B levels were elevated in naswar users as compared to controls.
\end{abstract}

KEYWORDS: Inflammation, Interleukin (IL-1B), Naswar, Periodontitis, Saliva.

How to cite this:

doi: https://doi.org/10.12669/pjms.35.3.10

Abbasi ZA, Hadi NI, Zubairi AM, Hosein M. Salivary Interleukin 1-beta levels and clinical periodontal parameters in habitual naswar users and non-users. Pak J Med Sci. 2019;35(3):674-679. doi: https://doi.org/10.12669/pjms.35.3.10

This is an Open Access article distributed under the terms of the Creative Commons Attribution License (http://creativecommons.org/licenses/by/3.0), which permits unrestricted use, distribution, and reproduction in any medium, provided the original work is properly cited.

\section{INTRODUCTION}

Naswar is categorized as one of the smokeless tobacco products (STPs), the ingredients of which are mainly sundried crushed local tobacco, ash, calcium oxide (slaked lime), and sometimes flavoring agents (e.g., cardamom, menthol) as well as coloring agents (indigo). ${ }^{1}$ It is commonly used in Pakistan, Afghanistan, Iran, Central Asia and South Africa. ${ }^{1}$ Naswar usage is gaining popularity as it is now available and being consumed in different parts of the world including England. ${ }^{2}$ It is prepared and wrapped into small plastic bags which even lack safety warnings. ${ }^{1}$ It is used mostly by applying and retaining it in the vestibular cavity adjacent to the buccal or labial mucosa or at times under the tongue. ${ }^{2}$ All the other types of STPs are consumed via chewing but naswar is never bitten because of its bad taste. ${ }^{2}$

The tobacco being currently used in naswar contains trace amounts of arsenic, beryllium, 
cadmium, chromium, cobalt, lead, nickel, nitrate, nitrite and tobacco specific nitrosamines (TSNAs). ${ }^{1}$ Along with these substances, addictive nicotine is also found in naswar. ${ }^{3}$

The main characteristics of chronic periodontitis are periodontal attachment loss, connective tissue degradation and resorption of alveolar bone. The disease process is initiated by bacterial pathogens however it has been proven that they alone cannot be responsible for the severity of tissue destruction occurring in periodontitis. ${ }^{4}$

Currently accepted theory related to pathogenesis of periodontitis suggests that alteration in host defenses by bacterial and host products stimulates the host inflammatory response, eventually causing tissue damage. ${ }^{5,6}$ Proinflammatory cytokines are considered to be important modulators of inflammation during the initiation and progression of periodontal disease. ${ }^{7,8}$ Interleukin (IL)-1, which is a proinflammatory cytokine plays a vital role in immune regulation that causes a variety of inflammatory responses. It has been identified as a periodontal disease marker because of its function as not only an inflammatory mediator but also as a modulator of extracellular matrix and bone. ${ }^{9}$ Although both types of IL-1 (IL-1a and IL-1 $\beta$ ) have similar biologic functions, IL-1 $\beta$ is more effective in stimulating bone resorption and occurs more often in periodontitis. ${ }^{10}$

Use of smokeless tobacco products such as naswar is widespread in the population of Karachi, contributing to inflammatory, premalignant and malignant diseases of the oral cavity. To the best of our knowledge the effect of naswar on periodontal health in terms of salivary IL-1 $\beta$ levels has not been studied so far. Hence, IL-1 $\beta$ levels in association with clinical periodontal parameters will give a better understanding of the effects of naswar, specifically on the periodontium.

The objectives of our study were first to assess the clinical periodontal parameters and salivary IL$1 \beta$ levels in naswar users and non-users and then to find a correlation of salivary IL- $1 \beta$ levels with clinical periodontal parameters.

\section{METHODS}

This study was conducted between August 2017 and May 2018. Convenience sampling technique was used for recruitment of participants from the dental outpatient department (OPD) of Ziauddin College of Dentistry, Ziauddin University, Karachi, Pakistan. A WHO Software ("Sample size determination in Health Sciences") was used for sample size calculation using $0.2 \%$ prevalence of naswar use as reported by Sajid and Bano (2016) at $95 \%$ confidence interval and $80 \%$ power of study. ${ }^{11}$ This was a cross-sectional study with eighty-four participants, which were divided into two equal groups. Both genders were included with age's $\geq$ 18 years. Individuals who reported consuming naswar daily for $\geq 1$ year were defined as naswar users (Group-1). Controls included those who had never used tobacco in any form (Group-2). Individuals who were present or former users of any other type of tobacco or alcohol products; had systemic inflammatory diseases that could affect the periodontal condition; antibiotics and antiinflammatory medication usage within 6 months or those who had any periodontal and/or orthodontic procedure in the past 6 months were excluded from the study.

This study was approved by the Ethical Review Committee (ERC) of Ziauddin University, Karachi, Pakistan (Reference code \# 0300917ZAOP). It was obligatory for all participants to sign an informed written consent in order to take part in this study.

A questionnaire was used to obtain data from all the participants regarding age, gender, ethnicity, educational status, habits related to naswar usage (time period since using, daily frequency, duration of holding a single bolus in the mouth, reason and location of naswar placing), oral hygiene practices and whether they considered naswar safe for consumption.

For collection of unstimulated whole saliva (UWS) samples, participants were asked to come in early morning hours. They were asked to refrain from eating or drinking at least two hours before saliva collection. For collection of saliva, the patient was first seated on a dental chair and asked to allow UWS to collect in the mouth for five minutes, which was then passively drooled into a graded plastic funnel tube. The samples were stored in a container filled with dry ice and immediately shifted to the Research Laboratory, Ziauddin University. Saliva samples were centrifuged at $2500 \mathrm{rpm}$ (Gemmy Industrial Corp., USA) for 20 minutes at $4^{\circ} \mathrm{C}$ and the supernatant (around $3 \mathrm{ml}$ ) was stored at $-80^{\circ} \mathrm{C}$ in multiple aliquots.

A trained examiner (ZA) performed the clinical periodontal examination. The overall kappa for intra examiner reliability was 0.84 . Full-mouth PI (plaque index), BOP (bleeding on probing), PD (probing depth) and CAL (clinical attachment loss) were measured at six sites per tooth (mesial, middle and 
distal points on buccal/labial and lingual/palatal surfaces) on all teeth of both arches (excluding third molars).$^{12}$ A University of North Carolina graded probe (Hu Friedy-15) was used to measure PD to the nearest millimeter.

For the quantitative determination of IL-1 $\beta$ concentration in saliva, (Glory Science Co., Ltd) human IL-1 $\beta$ enzyme linked immunosorbent assay (ELISA) kit was used. Manufacturer's instructions were followed for the indirect ELISA technique being performed. A spectrophotometer (Plate reader stat, Fax-2100, Awareness technology, USA) was used to determine the absorbance by reading the plates at $450 \mathrm{~nm}$. This was done twice to check for reproducibility of the results. The detection limit of the kit was 1-20ng/L.

Statistical Package for the Social Sciences (SPSS version 20, Chicago, IL) was used for statistical analysis. After checking normality using ShapiroWilk's test, Mann-Whitney test was run to compare PD, CAL, BI, and PI between naswar dippers and controls. Spearman's correlation was computed to find the correlation between IL$1 \beta$ with PD, CAL, BI and PI. A pValue less than .05 was set to show statistical significance of the findings.

\section{RESULTS}

A total of eighty-four male subjects were included in the study (forty-two naswar users and fortytwo healthy controls). Only two females reported occasional naswar usage instead of habitual, hence they were excluded from the study. The mean age of naswar users and controls were $33.7 \pm 10.8$ and $36.4 \pm 4.9$ years, respectively (Table-I). Naswar dippers mostly belonged to the Pathan community $(76.2 \%)$. Regarding educational status in naswar users and non-users, 59.5\% and 36.4\% were uneducated, respectively. Most common reason for using naswar was that they were just addicted to it $(52.4 \%)$ (Table-I). About $81.1 \%$ reported placing it in the premolar-molar vestibular area (right, left or in combination). The mean duration of habit was 12.8 \pm 8.1 years, whereas the mean duration of keeping a single naswar bolus was $7.2 \pm 4.9$ minutes (Table-I). An alarming $85.7 \%$ of the naswar dippers claimed that they considered naswar safe for consumption as it had no deleterious effect on the body, particularly oral cavity.

In maintaining oral hygiene, participants reported using brush, miswak and finger (with paste or tooth powder) for cleaning their teeth. Among naswar dippers, 35.7\% reported brushing and $40.5 \%$ miswak use at least once a day (Table-I). It is pertinent to mention that in our part of the world use of Miswak is very popular as a tool for cleaning the teeth. Brushing habit in naswar users and controls were $35.7 \%$ and $40.4 \%$, respectively. Use of miswak was found to be almost equivalent to brushing habits, that is in naswar users and controls it was $40.4 \%$ and $38.2 \%$, respectively. About $23.9 \%$ of naswar dippers and $21.4 \%$ of controls reported that they did not use any technique for maintaining oral hygiene (Table-I).

CAL, PD of $4 \mathrm{~mm}$ and in $5-6 \mathrm{~mm}$ range was significantly higher in naswar dippers than controls. Difference in clinical parameters among

Table-I: Characteristics of the study population.

\begin{tabular}{|c|c|c|}
\hline Characteristics & $\begin{array}{c}\text { Naswar- } \\
\text { dippers } \\
(n=42)\end{array}$ & $\begin{array}{c}\text { Controls } \\
(n=42)\end{array}$ \\
\hline Mean age (years) & $33.7 \pm 10.8$ & $36.4 \pm 4.9$ \\
\hline \multicolumn{3}{|l|}{ Ethnicity: } \\
\hline Pathans & $76.2 \%$ & $61.9 \%$ \\
\hline Sindhi & $21.4 \%$ & $21.4 \%$ \\
\hline Punjabi & $2.4 \%$ & $11.9 \%$ \\
\hline Urdu speaking/Muhajir & $0 \%$ & $4.8 \%$ \\
\hline \multicolumn{3}{|l|}{ Education status: } \\
\hline Undergraduate level & $39.5 \%$ & $58.9 \%$ \\
\hline Graduate level & $1 \%$ & $4.7 \%$ \\
\hline Uneducated & $59.5 \%$ & $36.4 \%$ \\
\hline \multicolumn{3}{|l|}{ Reason for using naswar: } \\
\hline Just addicted to it & $52.3 \%$ & \\
\hline Reduce psychological stress & $38.1 \%$ & NA \\
\hline For fresh breathe & $4.8 \%$ & \\
\hline For the taste & $4.8 \%$ & \\
\hline \multicolumn{3}{|l|}{$\begin{array}{l}\text { Position of placement of } \\
\text { naswar: }\end{array}$} \\
\hline Left buccal vestibule & $31 \%$ & \multirow{4}{*}{ NA } \\
\hline Right buccal vestibule & $19 \%$ & \\
\hline Both buccal vestibules & $31 \%$ & \\
\hline Adjacent to the lower lip & $19 \%$ & \\
\hline Duration of habit (years) & $12.8 \pm 8.1$ & NA \\
\hline $\begin{array}{l}\text { Mean daily frequency of } \\
\text { naswar-dipping }\end{array}$ & $20.3 \pm 10.6$ & NA \\
\hline $\begin{array}{l}\text { Mean duration of keeping a } \\
\text { single bolus (minutes) }\end{array}$ & $7.2 \pm 4.9$ & NA \\
\hline \multicolumn{3}{|l|}{ Oral hygiene maintenance: } \\
\hline $\begin{array}{l}\text { Brushing (at least once } \\
\text { daily) }\end{array}$ & $35.7 \%$ & $40.4 \%$ \\
\hline Miswak (at least once daily) & $40.4 \%$ & $38.2 \%$ \\
\hline None & $23.9 \%$ & $21.4 \%$ \\
\hline
\end{tabular}


Table-II: Mean and range of clinical periodontal parameters among naswar dippers and control group.

\begin{tabular}{lcc}
\hline & $\begin{array}{c}\text { Naswar- } \\
\text { dippers }\end{array}$ & Controls \\
\hline $\begin{array}{l}\text { Salivary IL-1 } \beta \\
(\mathrm{ng} / \mathrm{L})\end{array}$ & $8.3 \pm 3.1^{*}$ & $2.3 \pm 1.1$ \\
PD 4mm (\%) & $9.3(0.0-42.9)^{*}$ & $4.4(0.0-32.6)$ \\
PD 5-6mm (\%) & $5.0(0.0-33.9)^{*}$ & $2.3(0.0-28.3)$ \\
PD >=7mm (\%) & $0.1(0.0-1.6)$ & $0.3(0.0-8.0)$ \\
CAL (mm) & $2.9(1.3-5.7)^{* *}$ & $2.1(1.0-7.6)$ \\
Plaque Index (\%) & $3.1(0.0-13.4)$ & $2.6(0.0-9.1)$ \\
Bleeding Index (\%) & $2.7(0.0-11.6)$ & $2.4(0.0-11.1)$ \\
Missing teeth & $11.1(0.0-48.0)$ & $5.5(0.0-42.0)$ \\
\hline
\end{tabular}

*P-value $<0.05,{ }^{* *} \mathrm{P}$-value $<0.001$

Mann-Whitney U test applied.

the two groups was determined by applying Mann-Whitney $U$ test. There was no significant difference in PI, BI, PD $>7 \mathrm{~mm}$ and the number of missing teeth (Table-II).

Mean Interleukin 1- $\beta$ levels in naswar dippers and controls was $8.3 \pm 3.1 \mathrm{ng} / \mathrm{L}$ and $2.3 \pm 1.1 \mathrm{ng} / \mathrm{L}$, respectively (Table-II). There was a significant positive correlation between values of CAL $(\mathrm{P}<0.01)$ $\mathrm{PD} 4 \mathrm{~mm}$ and $5-6 \mathrm{~mm}(\mathrm{P}<0.05)$ with salivary Interleukin 1- $\beta$ levels after applying Spearman's correlation test (Table-III).

\section{DISCUSSION}

To the best of our knowledge after going through published literature, this is the first study assessing salivary IL-1 $\beta$ level in naswar users. Multiple studies have shown that use of STPs including gutka, snus, shammah etc, have harmful effects on the oral cavity eventually leading to malignant lesions. ${ }^{13,14}$ Studies show that STPs use among Pakistani population is more in males then in females, and there is even a larger difference among the rural population. ${ }^{15,16}$ As mentioned before the use of naswar has become widespread and is gaining popularity, therefore researchers are now focusing on studies reporting the effects of naswar on the oral cavity. A study assessing cytomorphometric changes in buccal smear of naswar users, reported dysplastic changes in the oral mucosa, hence pointing to the potential of causing oral premalignant/malignant lesions as well. ${ }^{17}$ Recent studies have also associated naswar usage with alveolar bone loss and concurrent aggravation of clinical periodontal parameters. ${ }^{13,18}$ Contrary to our findings, a study assessed IL-1 $\beta$
Table-III: Correlation of Interleukin 1- $\beta$ levels with clinical periodontal parameters.

\begin{tabular}{|c|c|c|}
\hline & \multicolumn{2}{|c|}{ IL-1 $\beta$ level } \\
\hline & $\begin{array}{c}\text { Correlation } \\
\text { value }\end{array}$ & $P$-value \\
\hline PD 4mm (\%) & $0.248^{*}$ & 0.023 \\
\hline PD 5-6mm (\%) & $0.254^{*}$ & 0.020 \\
\hline $\mathrm{PD}>=7 \mathrm{~mm}(\%)$ & 0.131 & 0.236 \\
\hline $\begin{array}{l}\text { Clinical attachment } \\
\text { loss }(\mathrm{mm})\end{array}$ & $0.332^{* *}$ & 0.002 \\
\hline Plaque Index (\%) & -0.072 & 0.515 \\
\hline Bleeding Index (\%) & -0.027 & 0.805 \\
\hline
\end{tabular}

${ }^{*} \mathrm{P}$-value $<0.05,{ }^{* *} \mathrm{P}$-value $<0.005$

Spearman's correlation test applied.

levels in serum of naswar dippers which showed the levels to be significantly lowered in naswar users as compared to healthy controls. ${ }^{11}$

Present study showed that IL-1 $\beta$ level were raised in the saliva of naswar users as compared to the healthy controls.IL-1 $\beta$ levels in both GCF and saliva of other type of STP users, was also found to be raised; emphasizing the point that IL$1 \beta$ plays a critical role in periodontitis and that its levels were increased more when associated with a risk factor such as use of STPs. ${ }^{14,19,20}$ This could be due to the fact that naswar users have a poorer periodontal condition as shown by elevated PD and CAL. An interesting finding in our study was that though there was very significant difference in CAL and PD of naswar users as compared to nonusers, the alteration in PI, BOP and missing number of teeth wasn't statistically significant. This could be attributed to the fact that most of the study population belonged to low socioeconomic status and were mostly uneducated; hence there was poor oral hygiene maintenance in the healthy controls as well. Similarly a justification for an increased number of missing teeth could be due to these patients opting for extraction rather than costly but appropriate treatments such as fillings and root canals.

GCF is the primary source of periodontitisassociated cytokines and host-derived enzymes; however these substances will ultimately enter into the saliva. ${ }^{21,22}$ Though, mostly GCF is taken as a sample medium, it is very difficult because:

1. It is a technique sensitive method, as collection requires skilled person who can prevent contamination of the GCF strips or paper points from saliva; 
2. There are chances of bleeding when collecting GCF which again contaminates the sample;

3. GCF strips or paper points require an added electronic Periotron device to determine the volume obtained, which is not available easily;

4. Collecting GCF through micropipette may cause trauma to the gingiva as well. In addition to these, saliva collection is less troublesome and several samples can be collected without causing any distress to the patient, hence it was the selected medium for this study. ${ }^{21}$

In this study we attempted to see the use of naswar among various ethnicities residing in Karachi city namely Urdu speaking/Muhajir, Punjabi, Sindhi and Pathans as previously naswar dipping was considered to be specific to those belonging from Pathan ethnic group. Though they are still the most common users of the product, a rise in usage is seen in other communities as well which is also shown in other studies as well. ${ }^{15}$

It is important for health care providers to have adequate knowledge regarding the detrimental effects of naswar usage so they can convey that knowledge to those who use this STP. There's an alarming myth among the naswar dippers that it is safe for consumption as compared to other STPs and has no detrimental effects on the oral cavity or to the body internally. Only $14.3 \%$ of the naswar users in our study believed that it may have detrimental effects on the oral tissues and may cause oral cancer. Due to increase in evidence of potential harmful effects of naswar, there is a need for the health department to take action against use of naswar, as they have recently imposed sanctions on sale of gutka and betel nuts in the country.

Limitation of the study: Answers to the questions regarding demographics and systemic illnesses were based on self-reporting and no test/ examination was done to actually determine if the participants were systemically healthy or not. This is a limitation of the study, as systemic diseases can have adverse effects on the periodontium and alter IL-1 $\beta$ levels as well.

\section{CONCLUSION}

Within the perimeters of this study, it can be concluded that IL-1 $\beta$ levels in saliva are raised and clinical periodontal conditions are worse in habitual naswar users as compared to non-users.
Future Recommendation: Levels of IL-1 $\beta$ might be checked both in saliva and GCF of naswar users, thus drawing a comparison between the effectiveness of the two mediums.

Acknowledgement: The authors are thankful to the Board of Advance Studies and Research (BASR), Ziauddin University for funding this research.

Deceleration of Interests: The authors declare that they have no competing interests.

Grant Support \& Financial Disclosures: Grant support from Board of Advanced Studies and Research (BASR), Ziauddin University.

\section{REFERENCES}

1. Zakiullah, Saeed M, Muhammad N, Khan SA, Gul F, Khuda $\mathrm{F}$, et al. Assessment of potential toxicity of a smokeless tobacco product (naswar) available on the Pakistani market. Tob. Control. 2012;21(4):396-401. doi 10.1136/tc.2010.042630.

2. Basharat S, Kassim S, Croucher R. Availability and use of Naswar: an exploratory study. J Public Health. 2012;34(1):6064. doi: $10.1093 /$ pubmed/fdr044.

3. Tomar SL. Is use of smokeless tobacco a risk factor for cigarette smoking? The U.S. experience. Nicotine Tob Res. 2003;5(4):561-569.

4. Delima AJ, Karatzas S, Amar S, Graves DT. Inflammation and tissue loss caused by periodontal pathogens is reduced by interleukin-1 antagonists. J Infect Dis. 2002;186(4):511516. doi 10.1086/341778

5. Bartold PM, Van Dyke TE. Periodontitis: a host-mediated disruption of microbial homeostasis. Unlearning learned concepts. Periodontol 2000. 2013;62(1):203-217. doi: 10.1111/j.1600-0757.2012.00450.x.

6. Cekici A, Kantarci A, Hasturk H, Van Dyke TE. Inflammatory and immune pathways in the pathogenesis of periodontal disease. Periodontol 2000. 2014;64(1):57-80. doi: 10.1111/ prd.12002.

7. Cetinkaya B, GuzeldemirE, Ogus E, BulutS. Proinflammatory and Anti-Inflammatory Cytokines in Gingival Crevicular Fluid and Serum of Patients With Rheumatoid Arthritis and Patients With Chronic Periodontitis. J Periodontol. 2013;84:84-93. doi: 10.1902/jop.2012.110467.

8. Yamazaki K, Honda T, Oda T, Ueki-Maruyama K, Nakajima $\mathrm{T}$, Yoshie $\mathrm{H}$, et al. Effect of periodontal treatment on the C-reactive protein and proinflammatory cytokine levels in Japanese periodontitis patients. J Periodontal Res. 2005;40(1):53-58. doi: 10.1111/j.1600-0765.2004.00772.x.

9. Boch JA, Wara-aswapati N, Auron PE. Concise Review Biological: Interleukin 1 Signal Transduction- Current Concepts and Relevance to Periodontitis. J. Dent. Res. 2001;80(2):400-407. doi: 10.1177/00220345010800020101.

10. Gupta G. Gingival crevicular fluid as a periodontal diagnostic indicator- II: Inflammatory mediators, hostresponse modifiers and chair side diagnostic aids. J Med Life. 2013;6(1):7-13.

11. Sajid F, Bano S. Pro inflammatory interleukins and thyroid function in Naswar (dipping tobacco) users: a case control study. BMC Endocr Disord. 2016;16:47. doi: 10.1186/s12902016-0127-5.

12. Armitage GC. Periodontal diseases: diagnosis. Ann Periodontol. 1996;1(1):37-215. 
13. Akram Z, Abduljabbar T, Hosain M, Al-Sowygh ZH, AlHamoudi N, Vohra F, et al. Comparison of periodontal inflammatory parameters among habitual gutkachewers and naswar-dippers: a split-mouth retrospective clinical study. Acta Odontol Scand. 2018;76(2):141-147. doi: 10.1080/00016357.2017.1394489.

14. Javed F, Al-Kheraif AA, Al Amri MD, Mikami T, Vohra F, Warnakulasuriya S, et al. Periodontal parameters and whole salivary cytokine profiles among habitual gutka chewers and non-chewers. J Periodontol. 2015;86(5):689-695. doi: 10.1902/jop.2015.140556.

15. Imam SZ, Nawaz H, Sepah YJ, Pabaney AH, Ilyas M, Ghaffar S. Use of smokeless tobacco among groups of Pakistani medical students - a cross sectional study. BMC Public Health. 2007;7(1):231. doi: 10.1186/1471-2458-7-231

16. Sultan AN, Khan KA, Tabrez A, Rabia H. Smokeless tobacco use among adult patients who visited family practice clinics in Karachi, Pakistan. J Oral Pathol Med. 2009;38(5):416-421. doi: 10.1111/j.1600-0714.2009.00754.x.

17. Masood R, Rozina NZ, Khola J, Khan A, Javed M, Raza A, et al. Cytomorphometry of buccal smears of Nicotiana tabacum (Naswar) users: A Pilot Study. Pak Oral Dental J. 2014;34:445-448.

18. Daood U, Abduljabbar T, Al-Hamoudi N, Akram Z. Clinical and radiographic periodontal parameters and release of collagen degradation biomarkers in naswar dippers. J Periodontal Res. 2018;53(1):123-130. doi: 10.1111/jre.12496.

19. Jacob PS, Nath S, Patel RP. Evaluation of interleukin-1 $\beta$ and 8 in gutka chewers with periodontitis among a rural Indian population. J Periodontal Implan. 2014;44(3):126-133. doi:10.5051/jpis.2014.44.3.126.
20. Gaphor SM, Ali SH, Abdullah MJ. Evaluation of Salivary Interleukin-1beta (IL-1 $\beta$ ) Level in Relation to the Periodontal Status in Smoker and Non-smoker Individuals. J Interdiscipl Med Dent Sci. 2014;2(2376-032X). doi:10.4172/2376032X.1000120.

21. Javaid MA, Ahmed AS, Durand R, Tran SD. Saliva as a diagnostic tool for oral and systemic diseases. J Oral Biol Craniofac Res. 2016;6(1):66-75. doi:10.1016/j. jobcr.2015.08.006

22. Taylor JJ. Protein Biomarkers of Periodontitis in Saliva. ISRN Inflamm. 2014;18. doi: 10.1155/2014/593151.

\section{Author`s Contribution:}

NIH \& MH conceived, designed \& editing of manuscript.

ZAA did data collection, statistical analysis and manuscript writing.

ZAA \& AMZ handled the saliva collections \& testing.

NIH, MH \& AMZ did review and final approval of manuscript. 